\title{
INVESTIGATION OF THE DEPENDENCE OF DIELECTRIC PROPERTIES OF D-SERINE ADMIXTURED TGS CRYSTALS ON THE DISTANCE FROM THE SEED OF THE CRYSTAL GROWTH
}

\section{J. Stankowska, A. Czarnecka and Bangobango Dongo Gemi}

Institute of Physics, A. Mickiewicz University, Grunwaldzka 6, 60-780 Poznań, Poland

(Received October 9, 1992; in final form January 12, 1993)

Dielectric properties of a D-serine admixtured triglycine sulphate (TGS) crystal were studied on samples cut out from different sites in the crystal. The value of spontaneous polarization $P_{s}$ measured on $b$-cuts was found to be independent of the site. Whereas the coercive field $E_{c}$ values were higher for the samples cut out closer to the seed than for those cut out at the ends of the crystal along $b$ axis. After rejuvenation the values of the bias field $E_{\mathrm{b}}$ increased in contrary to the effect observed in the pure TGS crystals. An exponentially decreasing dependence of the maximum permittivity $\varepsilon_{\max }$ on the site of the samples ( $b$-plates) cut out along $b$ axis from $-b$ to $+b$ positions, was obtained.

PACS numbers: $77.22 . \mathrm{Ch}$

\section{Introduction}

An effective way of stabilization of one sense of the polarization vector in triglycine sulphate (TGS) crystals is introduction of an chiral organic (e.g. serine, alanine) [1-12] or inorganic (metal ion, e.g. chromium, iron, copper) [13-16] admixture to the solution from which the TGS crystals are grown. The influence of serine admixture on dielectric properties of the TGS crystals has been studied in $[8,9,15]$. The chemical formula difference between serine and glycine is that the hydrogen atom at the $\alpha$-carbon in serine is replaced by the group $\mathrm{CH}_{2} \mathrm{OH}$ in glycine. TGS crystals with D-serine admixture grow asymmetrically with respect to the seed along " $b$ " axis similarly as it was observed for L-serine TGS crystal, though the asymmetry was now found to develop in the opposite direction. Asymmetry of the same kind was also observed for TGS crystals admixtured with. $\mathrm{L}-\alpha$-alanine $[4,6,10,11]$ as well as $\mathrm{D}$ - $\alpha$-alanine [11]. 
It is known $[8,9]$ that with increasing concentration of serine admixture in the crystal:

- the maximum permittivity $\varepsilon_{\max }$ decreases,

- the values of the coercive field $E_{\mathrm{c}}$ and bias field $E_{\mathrm{b}}$ increase,

- the value of spontaneous polarization $P_{\mathrm{s}}$ decreases,

- the domain structure becomes more refined.

Nakatani $[5,6]$ has studied the distribution of the bias field in the TGS crystals admixtured with L- $\alpha$-alanine and DL- $\alpha$-alanine and has reported that $E_{\mathrm{b}}$ is not homogeneous within a growth pyramid and its values are higher on the $-b$ side than on the $+b$ side in accordance with Refs. $[10,12]$. The changes in the $E_{\mathrm{b}}$ values correspond to the changes in the alanine admixture concentration in the crystal.

The aim of this paper is to check the dependence of dielectric properties of the D-serine admixtured TGS (DSTGS) crystals on the site of the sample cut-out as well as to determine the distribution of the admixture in the crystal.

\section{Experimental}

The raw material of pure TGS crystals was subject to triple recrystallization [17] and the D-serine admixtured TGS crystals were grown from the solution containing $5 \mathrm{wt} . \%$ of D-serine admixture relative to dry mass of TGS.

The samples whose properties were measured were cut out from the pure TGS crystal grown in the ferroelectric phase at $318 \mathrm{~K}$ and from the DSTGS crystal grown at $316 \mathrm{~K}$. The samples of both kinds of crystals were cut out at different distances from the seeds which is shown schematically in Fig. 1. All (b-cuts) samples of $0.25-0.5 \mathrm{~cm}^{2}$ in area and of $0.1-0.2 \mathrm{~cm}$ thick were polished preserving the natural (001) wall of crystal (Fig. 1).
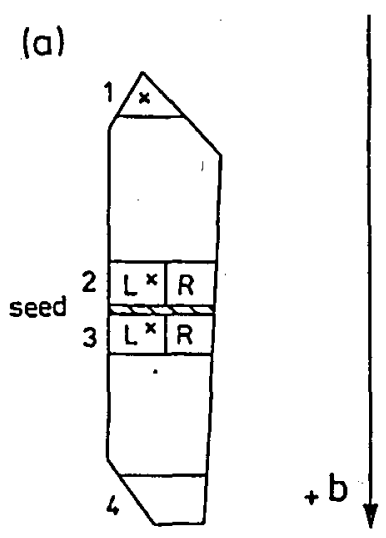

(b)

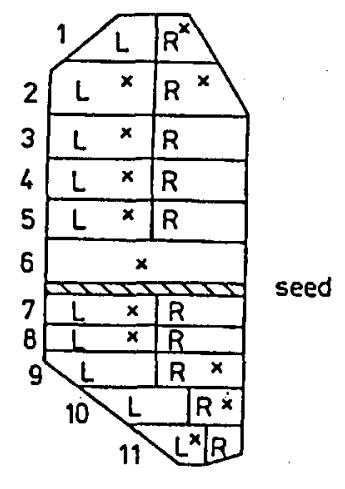

Fig. 1. A scheme of the crystal division into samples: (a) pure TGS, (b) D-serine admixtured TGS. L - left, $R$ - right sites of a crystal. $\times$ - the measured samples.

Silver electrodes were evaporated on the surface perpendicular to the $b$ axis. For each sample we measured spontaneous polarization $P_{\mathrm{s}}$, coercive field $E_{\mathrm{c}}$, bias 
field $E_{\mathrm{b}}$ and permittivity as a function of temperature $\varepsilon(T)$. Dielectric hysteresis loop measurements were carried out at room temperature by the Diamanta-Dvencka-Pepinsky (DDP) method applying an electric field of $290 \mathrm{kV} / \mathrm{m}$. Temperature measurements of permittivity $\varepsilon(T)$ were performed using an E-317 type RLC bridge operating at a frequency of $1 \mathrm{kHz}$ and a measuring field of $0.7 \mathrm{~V} / \mathrm{m}$. In the vicinity of the Curie point the rate of temperature changes was $0.1-0.2 \mathrm{~K} / \mathrm{min}$ both on heating and on cooling.

The error of measurement was $5 \%$ for permittivity determination, $6 \%$ for spontaneous polarization, $7 \%$ for coercive field and bias field. We measured old (aged) and rejuvenated (young) samples. The samples of the DSTGS crystal were rejuvenated by annealing at about 348-368 $\mathrm{K}$ for 48 hours while the samples of the pure TGS crystal were rejuvenated by annealing at about $338 \mathrm{~K}$ for 24 hours.

\section{Results}

\subsection{Permittivily measurements}

Tables I and II present the measured values of dielectric parameters obtained for the pure TGS crystal (Tab. I) and the TGS+D-serine crystal (Tab. II).

\section{TABLE I}

The values of the Curie temperatures $T_{\mathrm{C}}, \Delta T_{\mathrm{C}}$ and maximum permittivity $\varepsilon_{\max }, \Delta \varepsilon_{\max }$ obtained for the pure TGS crystals for aged samples on heating and for rejuvenated samples on cooling, $L$ means left sites of a crystal.

\begin{tabular}{l|c|c|c|r|r|r}
\hline \hline \multirow{2}{*}{ No } & \multicolumn{2}{|c|}{$T_{\mathrm{C}}[\mathrm{K}]$} & \multirow{2}{*}{$\Delta T_{\mathrm{C}}$} & \multicolumn{2}{|c|}{$\varepsilon_{\max }\left(10^{3}\right)$} & $\Delta \varepsilon_{\max }$ \\
\cline { 2 - 3 } \cline { 5 - 6 } & Aged & Young & {$[\mathrm{K}]$} & Aged & Young & $\left(10^{3}\right)$ \\
\hline 1 & 322.60 & 322.16 & 0.44 & 34.9 & 275.3 & 239.2 \\
$2 \mathrm{~L}$ & 322.00 & 321.65 & 0.65 & 33.5 & 121.2 & 87.7 \\
\hline \multicolumn{7}{c}{ Seed } \\
\hline 3 L & 321.28 & 321.13 & 0.15 & 23.8 & 141.5 & 117.7 \\
4 & 322.28 & 322.15 & 0.13 & 7.4 & 210.8 & 203.4
\end{tabular}

Figure 2 presents the temperature dependence of permittivity for three rejuvenated samples cut out from three different sites (numbered 1,6,11) in a TGS crystal admixtured with $5 \%$ of D-serine. The values of the maximum permittivity, $\varepsilon_{\max }$, decrease when passing from the extreme site of " $-b$ " (sample no. 1) to " $+b$ " (sample no. 11). We use the setting of crystallographic axes adopted by Březina, Havránková [11] and Nakatani [6].

Figures $3 \mathrm{a}$ and $3 \mathrm{~b}$ present the maximum permittivity values against the sites of the $b$-cuts samples, cut out in the sequence along the $b$ axis (Fig. 1) for aged and rejuvenated samples of the pure TGS (Fig. 3a) and DSTGS crystals (Fig. 3b) on cooling. The maximum permittivity values are found to decrease exponentially 
TABLE II

The values of the Curie temperatures $T_{\mathrm{C}}, \Delta T_{\mathrm{C}}$, and the values of maximum permittivity $\varepsilon_{\max }, \Delta \varepsilon_{\max }$ as well as the values of permittivity at room temperature $\varepsilon_{\mathbf{r}}$ (rejuvenated samples) obtained for D-serine admixtured TGS crystal for aged samples on heating and for rejuvenated on cooling, $\mathrm{L}$ - left, $\mathrm{R}$ - right sites of a crystal.

\begin{tabular}{|c|c|c|c|c|c|c|c|}
\hline \multirow[t]{2}{*}{ No } & \multicolumn{2}{|c|}{$T_{\mathrm{C}}[\mathrm{K}]$} & \multirow{2}{*}{$\begin{array}{r}\Delta T_{\mathrm{C}} \\
{[\mathrm{K}]}\end{array}$} & \multicolumn{2}{|c|}{$\varepsilon_{\max }\left(10^{3}\right)$} & \multirow{2}{*}{$\begin{array}{c}\Delta \varepsilon_{\max } \\
\left(10^{3}\right)\end{array}$} & \multirow[t]{2}{*}{$\overline{\varepsilon_{\mathrm{r}}}$} \\
\hline & Aged & Young & & Aged & Young & & \\
\hline $1 \mathrm{R}$ & 322.21 & 322.60 & -0.39 & 13.0 & 28.25 & 15.5 & 60 \\
\hline $2 \mathrm{R}$ & 322.86 & 323.02 & -0.16 & 11.7 & 22.2 & 10.5 & 44 \\
\hline $2 \mathrm{~L}$ & 323.21 & 322.80 & -0.41 & 11.5 & 17.7 & 6.2 & - \\
\hline $3 \mathrm{~L}$ & 321.86 & 323.12 & -1.26 & 15.9 & 26.1 & 10.2 & 48 \\
\hline $4 \mathrm{~L}$ & 323.34 & - & - & 8.9 & 17.5 & 8.6 & 44 \\
\hline $5 \mathrm{~L}$ & 323.45 & 323.25 & 0.20 & 8.5 & 19.7 & 11.2 & 52 \\
\hline 6 & 321.58 & 322.82 & -1.24 & 8.9 & 14.7 & 5.8 & 42 \\
\hline \multicolumn{8}{|c|}{ Seed } \\
\hline $7 \mathrm{~L}$ & 323.01 & 323.00 & 0.01 & 10.3 & 16.4 & 6.1 & 47 \\
\hline $8 \mathrm{~L}$ & 323.03 & 322.71 & 0.34 & 5.2 & 8.3 & 3.2 & 36 \\
\hline $9 \mathrm{R}$ & 322.32 & 323.23 & -0.91 & 12.3 & 16.5 & 4.2 . & - \\
\hline $10 \mathrm{R}$ & 321.82 & 322.32 & -0.50 & 6.6 & 15.3 & 8.7 & 42 \\
\hline $11 \mathrm{~L}$ & 323.02 & 323.23 & -0.21 & 8.3 & 9.3 & 1.0 & 42 \\
\hline
\end{tabular}

when passing from $-b$ to $+b$ along the $b$ axis for all samples except those of the rejuvenated pure TGS crystal.

Figure 4 presents the dependence of the maximum permittivity on the bias field for the rejuvenated D-serine admixtured TGS crystal. The samples characterized by the high bias field reveal low values of the maximum permittivity.

\subsection{Measurements of dielectric histeresis loop}

Tables III and IV present the results of measurements of spontaneous polarization, coercive field and bias field for aged and rejuvenated samples of the pure TGS (Table III) and DSTGS (Table IV) crystals cut out from different sites in the crystal.

Figures $5 \mathrm{a}$ and $5 \mathrm{~b}$ present the dependence of coercive field on the site of the samples for the aged and rejuvenated pure TGS (Fig. 5a) and DSTGS crystal (Fig. 5b). For the pure TGS crystal we find that the samples cut out close to the seed reveal lower values of coercive field than the samples cut out from the crystal ends. The situation is reversed for the DSTGS crystals whose samples cut out close to the seed show higher coercive field values than those cut out from the crystal 


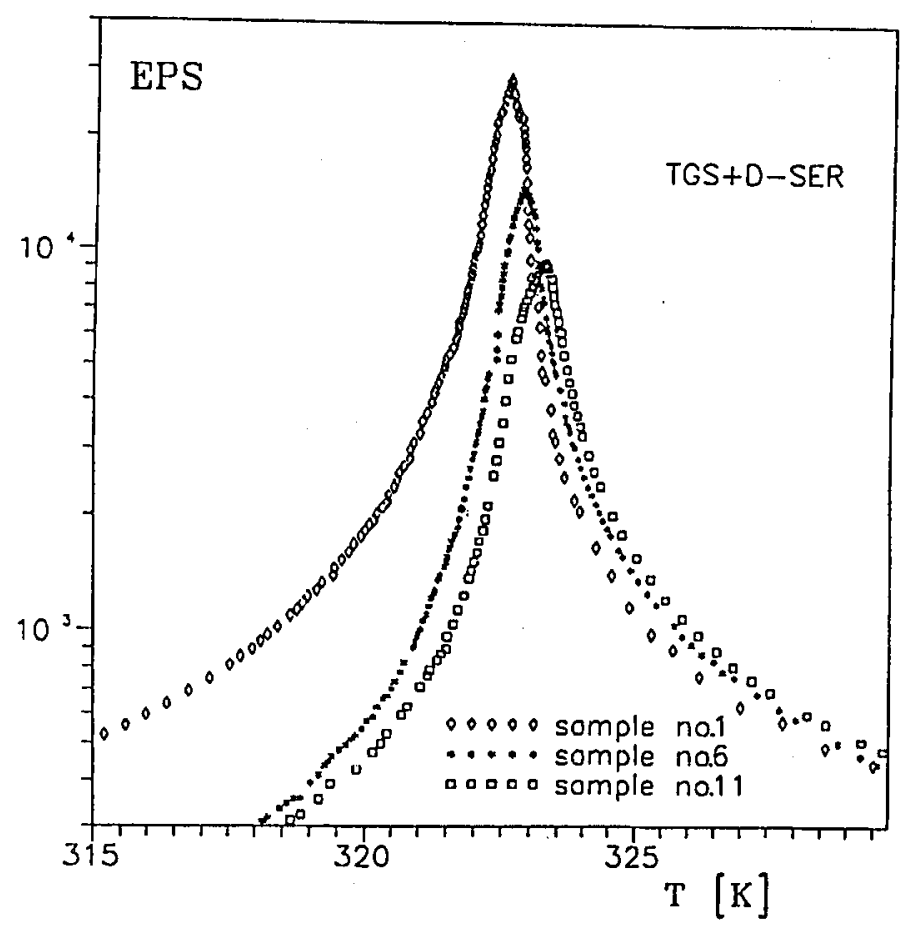

Fig. 2. Temperature dependence of permittivity for rejuvenated D-serine admixtured samples no. 1, 6, 11 .
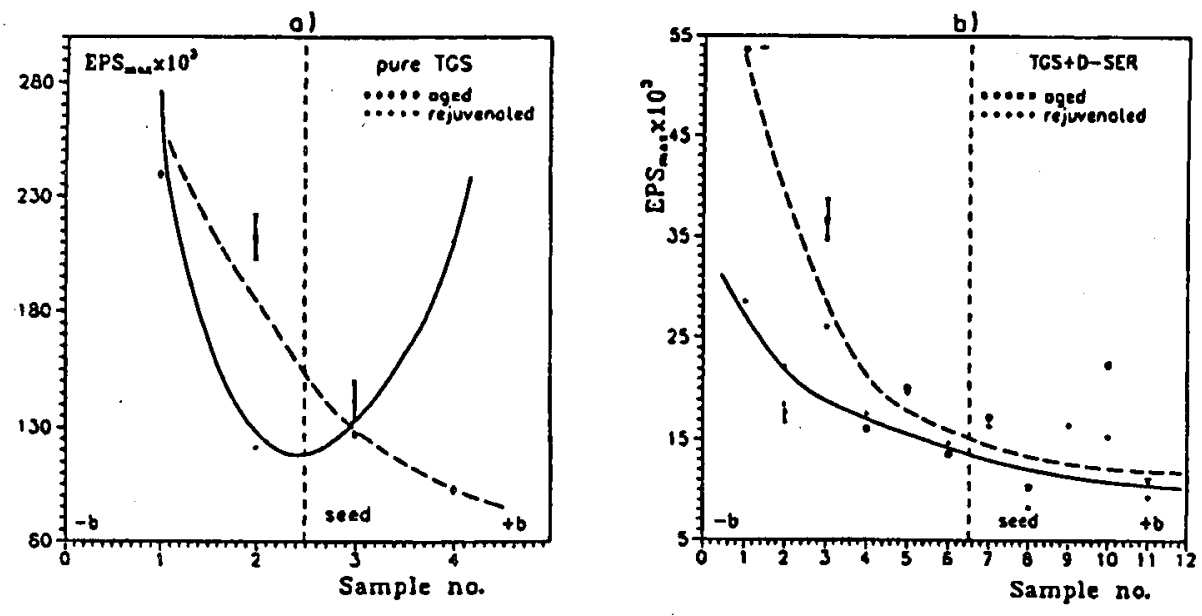

Fig. 3. The maximum permittivity values vs. the site of the samples for aged and rejuvenated samples of the pure TGS crystal (a) and D-serine admixtured TGS crystal (b), both obtained on cooling. 


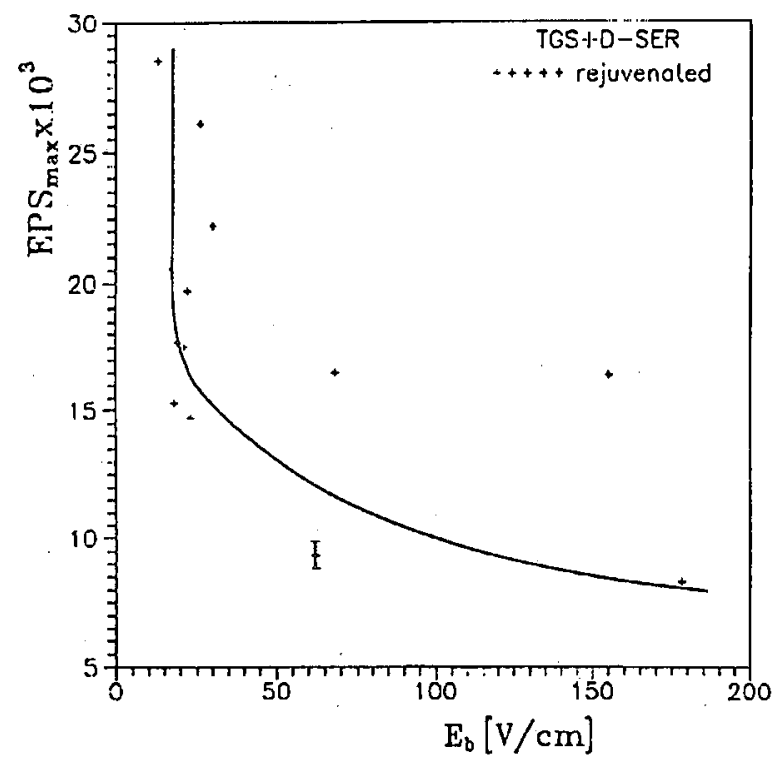

Fig. 4. The values of the maximum permittivity $\varepsilon_{\max }$ versus the bias field $E_{\mathrm{b}}$ for the rejuvenated D-serine admixtured crystal.

TABLE III

The values of the dielectric hysteresis loop parameters for the aged and rejuvenated samples of pure TGS cut out from different sites in the crystal, L left sites of a crystal.

\begin{tabular}{l|c|c|c|c|c|c}
\hline \hline \multirow{2}{*}{$\begin{array}{l}\text { Samples } \\
\text { number }\end{array}$} & \multicolumn{2}{c|}{$P_{\mathrm{s}}\left[\mu \mathrm{C} / \mathrm{cm}^{2}\right]$} & \multicolumn{2}{c|}{$E_{\mathrm{c}}[\mathrm{V} / \mathrm{cm}]$} & \multicolumn{2}{c}{$E_{\mathrm{b}}[\mathrm{V} / \mathrm{cm}]$} \\
\cline { 2 - 7 } & Old & Young & Old & Young & Old & Young \\
\hline 1 & 2.6 & 2.6 & 560 & 530 & 60 & 40 \\
$2 \mathrm{~L}$ & 2.8 & 2.7 & 500 & 490 & 32 & 23 \\
\hline \multicolumn{6}{c}{ Seed } \\
\hline $3 \mathrm{~L}$ & 2.8 & 2.8 & 500 & 460 & 30 & 21 \\
4 & 2.2 & 2.7 & 540 & 590 & 10 & 29
\end{tabular}

ends along the $b$ axis. After rejuvenation, in the pure TGS crystal the coercive field decreases whereas in the DSTGS crystal it increases.

Figure 6 illustrates the results of the bias field measurements for different sites of the sample cut out from the rejuvenated DSTGS crystal. The samples cut out on the $+b$ side show higher values of the bias field than those cut out on the $-b$ side. Similar results were obtained for the aged samples of the DSTGS crystals.

In Fig. 7 we illustrate the relationship between the coercive field and the bias field. As can be seen the coercive field increases with increasing bias field. 
TABLE IV

The values of the dielectric hysteresis loop parameters for the aged and rejuvenated D-serine admixtured TGS crystal cut out from different sites in the crystal, L - left, R - right sites of a crystal.

\begin{tabular}{c|c|c|c|c|r|c}
\hline \hline \multirow{2}{*}{$\begin{array}{c}\text { Sample } \\
\text { number }\end{array}$} & \multicolumn{2}{|c|}{$P_{\mathbf{s}}\left[\mu \mathrm{C} / \mathrm{cm}^{2}\right]$} & \multicolumn{2}{|c|}{$P_{\mathrm{c}}[\mathrm{V} / \mathrm{cm}]$} & \multicolumn{2}{|c}{$P_{\mathrm{b}}[\mathrm{V} / \mathrm{cm}]$} \\
\cline { 2 - 7 } & Old & Young & Old & Young & Old & Young \\
\hline 1R & 2.7 & 3.0 & 520 & 534 & 30 & 13 \\
2R & 3.0 & 2.9 & 487 & 455 & 2 & 30 \\
2L & 2.9 & 3.0 & 493 & 599 & 13 & 19 \\
3L & 2.7 & 2.5 & 540 & 538 & 22 & 26 \\
4L & 2.4 & 2.8 & 487 & 566 & 6 & 21 \\
5L & 2.8 & 2.9 & 531 & 693 & 67 & 22 \\
6 & 2.7 & 3.2 & 592 & 595 & 8 & 23 \\
\hline \multicolumn{7}{|c}{ Seed } \\
\hline 7L & 2.2 & 3.1 & 514 & 642 & 44 & 155 \\
8L & 2.4 & 2.8 & 533 & 618 & 209 & 178 \\
9R & 2.7 & 2.9 & 544 & 543 & 107 & 68 \\
10R & 2.7 & 3.2 & 490 & 469 & 25 & 18 \\
11L & 2.8 & 3.0 & 542 & 615 & 13 & 62
\end{tabular}
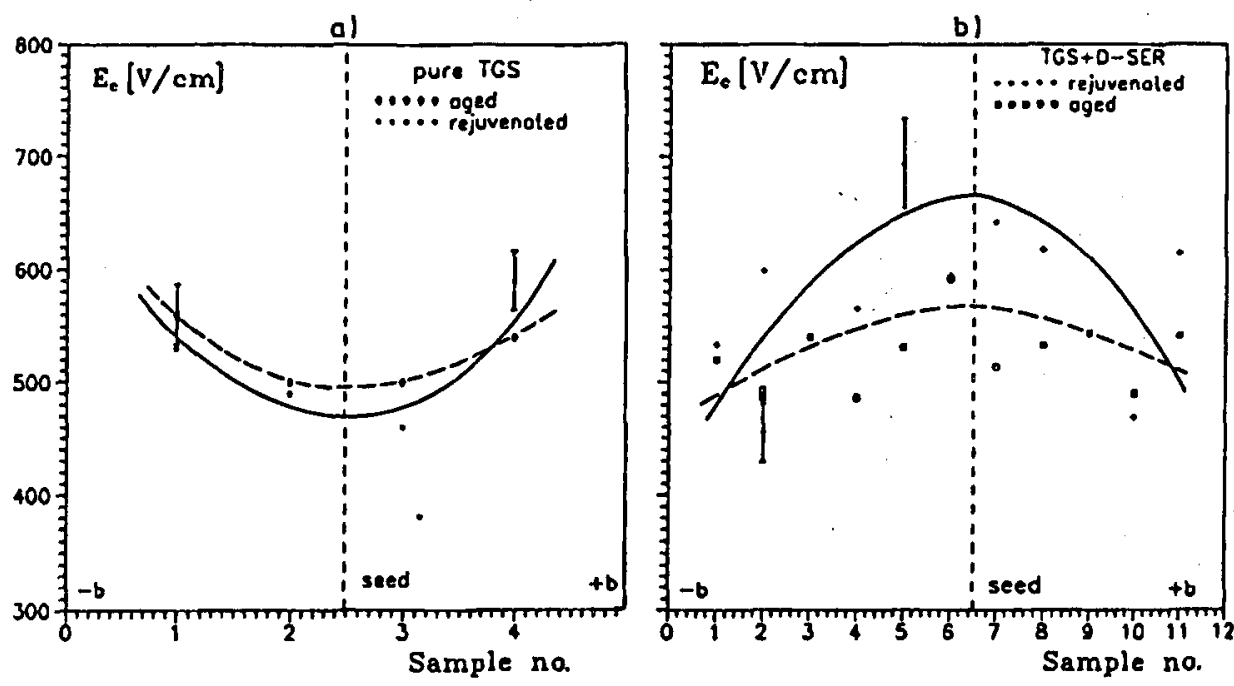

Fig. 5. The coercive field $E_{c}$ versus the site of the sample cut-out from the aged and rejuvenated pure TGS crystal (a) and D-serine admixtured TGS crystal (b). 


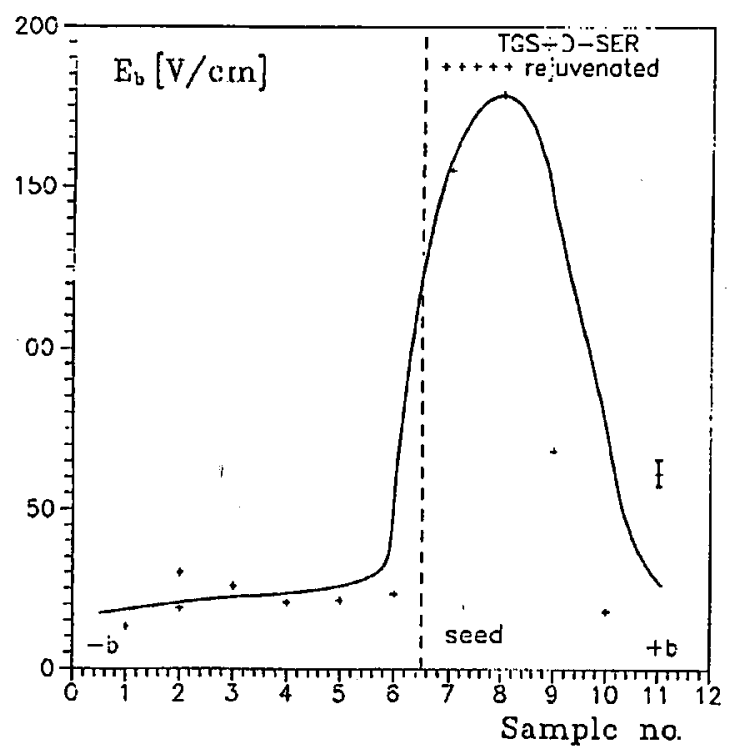

Fig. 6. The values of the bias field $E_{\mathrm{b}}$ for different sites of the sample cut-out from the rejuvenated D-serine admixtured crystals.

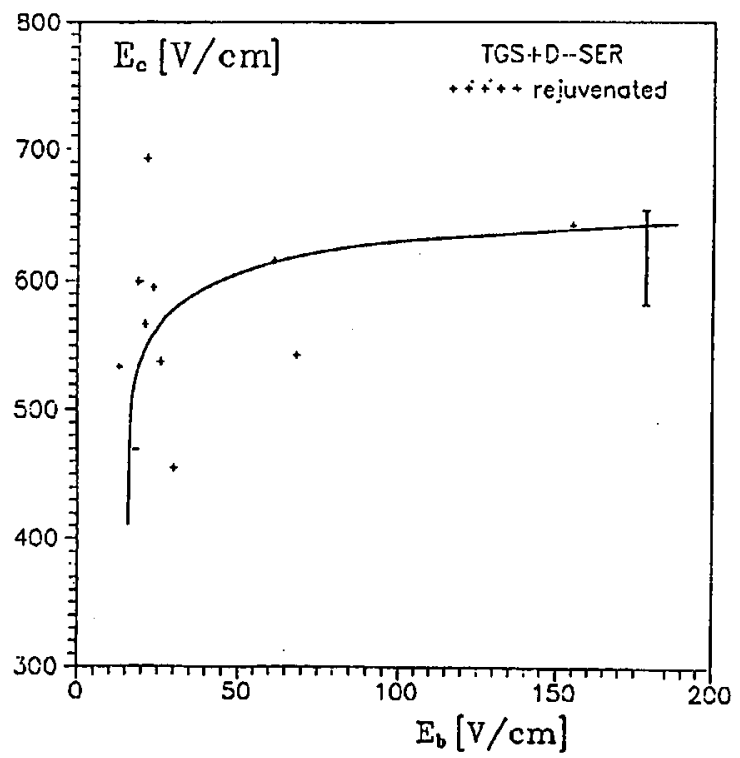

Fig. 7. The coercive field $E_{c}$ versus the bias field $E_{\mathrm{b}}$ for the rejuvenated D-serine admixtured crystals. 


\section{Discussion of the results and conclusions}

The obtained dependence of the maximum permittivity on the site of the sample cut-out, illustrated in Figs. 3a and 3b, is similar for the aged pure TGS crystal and DSTGS crystal, and can be described as a linear function decreasing from $-b$ to $+b$. After rejuvenation of the samples of pure TGS, a significant increase in the maximum permittivity, $\varepsilon_{\max }$ and $\Delta \varepsilon_{\max }$, is observed on the $+b$ side (and the obtained dependence is of the parabolic shape).

In the DSTGS crystals the dependence of the maximum permittivity on the site of the sample cut-out along the $b$ axis from $-b$ to $+b$ decreases. For the rejuvenated pure TGS crystal this dependence is of parabolic shape with the lowest values of the maximum permittivity measured for the samples cut out in the vicinity of the nucleus and higher for the samples cut out at the ends of the crystal. The maximum permittivity values are higher by one order of magnitude for the pure TGS crystals than for the DSTGS crystals. It may be supposed that $\mathrm{D}$-serine admixture stabilizes one sense of the polarization vector in a given crystal to a greater degree than it occurs in the process of spontaneous ageing.

In the pure TGS crystal the coercive field values are the lowest in the vicinity of the seed (Fig. 5a), whereas in the DSTGS crystal the coercive field values are the highest for the samples cut out close to the seed (Fig. 5b). The TGS crystals admixtured with L-serine and D-serine $[8,9]$ (similarly as those admixtured with $\mathrm{L}$ - $\alpha$-alanine $[4,7]$ or $\mathrm{D}-\alpha$-alanine [11]) grow asymmetrically with respect to the seed. In the case of D-serine TGS on the longer side $(-b)$ the maximum permittivity values are higher and the bias field values are lower than on the shorter side $(+b)$. This confirms that D-serine admixture is asymmetrically built into the crystal causing an inhibition of growth along the $b$ axis on the $+b$ side. Additional evidence supporting this conclusion is that a TGS crystal admixtured with DL-serine [18] grows symmetrically with respect to the seed. Similar results for LATGS crystals have been obtained by Březina, Havránková, Váša [19] and Nakatani [6].

The results of the measurements performed for the D-serine admixtured crystals allow us to draw the following conclusions:

1. The maximum permittivity values $\varepsilon_{\max }$ and $\Delta \varepsilon_{\max }$ decrease along the $b$ axis from $-b$ to $+b$.

2. The values of spontaneous polarization do not depend on the site of the sample cut-out.

3. The coercive field values are greater in the vicinity of the seed than at the ends of the crystal measured along the $b$ axis and increase after crystal rejuvenation.

4. The bias field values are greater on the $+b$ side than on the $-b$ side of the $b$ axis.

5. The $\mathrm{D}$-serine admixture is asymmetrically built into the crystal with respect to the seed with (010) planes, less admixture is build on the $-b$ side (longer part of the crystal) than on the $+b$ side (shorter one) of the $b$ axis.

The results of the measurements performed for the pure TGS crystal lead to the following conclusions: 
1. For the aged crystal the maximum permittivity values $\varepsilon_{\max }$ and $\Delta \varepsilon_{\max }$ are exponentially dependent on the site of the samples (b-cuts) cut out in various positions along the $b$ axis from $-b$ to $+b$.

2. Spontaneous polarization does not depend on the site of the sample cut-out.

3. The coercive field $E_{\mathrm{c}}$ is higher at the ends of the crystal along the $b$ axis than in the vicinity of the seed of growth, after rejuvenation its values decrease.

\section{References}

[1] P.J. Lock, Appl. Phys. Let. 19, 390 (1971).

[2] E.T. Keve, K.L. Bye, P.W. Whipps, D.A. Annis, Ferroelectrics 3, 39 (1971).

[3] J. Stankowska, A. Czarnecka, D. Tarnówka, Acta Phys Pol. A 76, 529 (1989).

[4] M. Koralewski, J. Stankowska, T. Jasiński, Jpn. J. Appl. Phys. 26, 383 (1987).

[5] N. Nakatani, Jpn. J. Appl. Phys. 30, 3445 (1991).

[6] N. Nakatani, Jpn. J. Appl. Phys. 30, 1024 (1991).

[7] S. Duka, V. Klubovich, N. Tolocho, N. Tikhomirova, L. Dontstova, Ferroelectr. Lettr. Sect. 10, 161 (1989).

[8] J. Stankowska, A. Czarnecka, G. Kwitowska, Ferroelectrics 108, 1931 (1990).

[9] J. Stankowska, A. Czarnecka, L. Dejneka, Izv. Akad. Nauk SSSR Ser. Fiz. 55, 496 (1991).

[10] B. Březina, M. Havránková, Cryst. Res. Technol. 20, 787 (1985).

[11] B. Březina, M. Havránková, Cryst. Res. Technol. 20, 781 (1985).

[12] B.I. Lilliczap, J.D.C. Wood, J. Cryst. Growth 41, 205 (1977).

[13] M. Tyoda, Y. Tanaka, W. Shioka va, Rev. Electr. Commun. Lab. 9, 485 (1961).

[14] F. Mora vec, J. Novotný, K'rist. Technik 6, 335 (1971).

[15] J. Stankowska, H. Kuś, Fizyka Dielektr. i Radiospektr. 10, 55 (1978) (in Polish).

[16] J. Stankowska, Acta Phys. Pol. 31, 527 (1967).

[17] F. Mora vec, Z. Malek, Z. Sulcek, J. Hrdlička, J. Phys. Soc. Jpn. 28, 434 (1970).

[18] J. Stankowska, A. Czarnecka, A. Dratwiniska, Ferroelectrics, in press.

[19] B. Březina, M. Havránková, M. Váša, Cryst. Res. Technol. 27, 13 (1992). 